\title{
Below Safety Limits, Every Unit of Glomerular Filtration Rate Counts: Assessing the Relationship Between Renal Function and Cancer-specific Mortality in Renal Cell Carcinoma
}

\author{
Alessandro Antonelli ${ }^{a,}{ }^{*}$, Andrea Minervini ${ }^{b}$, Marco Sandri $^{c}$, Roberto Bertini $^{d}$, \\ Riccardo Bertolo $^{e}$, Marco Carini $^{b}$, Maria Furlan $^{a}$, Alessandro Larcher $^{d}$, Guglielmo Mantica $^{f}$, \\ Andrea Mari $^{b}$, Francesco Montorsi $^{d}$, Carlotta Palumbo ${ }^{a}$, Francesco Porpiglia $^{e}$, \\ Paola Romagnani $^{g}$, Claudio Simeone $^{a}$, Carlo Terrone $^{f}$, Umberto Capitanio $^{d}$ \\ ${ }^{a}$ Department of Urology, Spedali Civili Hospital, University of Brescia, Brescia, Italy; ${ }^{\mathrm{b}}$ Department of Urology, Careggi Hospital, University of Florence, \\ Florence, Italy; ${ }^{\mathrm{c}}$ Data Methods and Systems Statistical Laboratory, University of Brescia, Brescia, Italy; ${ }^{\mathrm{d}}$ Division of Experimental Oncology, Department of

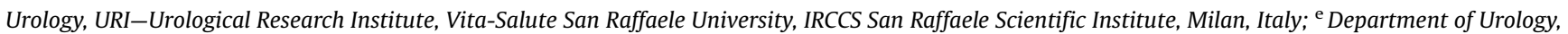 \\ San Luigi Gonzaga Hospital, School of Medicine, Orbassano, Turin, Italy; ${ }^{\mathrm{f}}$ Department of Urology, IRCCS San Martino Hospital, University of Genoa, Genoa, \\ Italy; ${ }^{\mathrm{g}}$ Department of Biomedical Experimental and Clinical Science "Mario Serio", University of Florence, Florence, Italy
}

\section{Article info}

Article history:

Accepted July 19, 2018

Associate Editor:

Giacomo Novara

Statistical Editor:

Andrew Vickers

\section{Keywords:}

Cancer-specific mortality Estimated glomerular filtration rate

Prognosis

Renal cell carcinoma

Renal function

Partial nephrectomy

Radical nephrectomy

\begin{abstract}
Background: The hypothesis that renal function could influence oncological outcomes is supported by anecdotal literature.

Objective: To determine whether estimated glomerular filtration rate (eGFR) is related to cancerspecific mortality (CSM) in patients who had undergone surgery for renal cell carcinoma (RCC).

Design, setting, and participants: A retrospective analysis of 3457 patients who underwent radical (39\%) or partial nephrectomy (61\%) for cT1-2 RCC between 1990 and 2015.

Outcome measurements and statistical analysis: The eGFR was calculated by the Chronic Kidney Disease Epidemiology Collaboration equation. CSM was analyzed in a multivariable competing-risk framework, estimating the subdistribution hazard ratio (SHR) accounting for deaths from other causes. The relationship between eGFR and CSM was investigated from multiple statistical approaches-extended Cox regression with eGFR incorporated as a time-dependent covariate, landmark analysis, and joint modeling. Other predictors were selected by competing-risk random forest method and backward elimination.

Results and limitations: The relationship between eGFR and CSM was graphically described by a linear spline, i.e. a continuous piecewise linear function with two lines joined by a knot. For eGFR treated as a time-dependent covariate, the knot was located at $65 \mathrm{ml} / \mathrm{min}$; at landmark analysis with eGFR at the baseline, $12 \mathrm{mo}$, and last functional follow-up, the knots were 85,60 , and $65 \mathrm{ml} / \mathrm{min}$, respectively. In multivariable competing-risk analysis, CSM was associated with eGFR only for values of eGFR below these cutoffs, with SHRs for every $10 \mathrm{ml} / \mathrm{min}$ of reduction in eGFR of $1.25(p=0.003)$, $1.16(p=0.028), 1.44(p=0.02)$, and $1.16(p=0.042)$, corresponding to time-dependent eGFR, and eGFR at baseline, $12 \mathrm{mo}$, and last functional follow-up, respectively. Joint modeling confirmed these results. A retrospective design with inherent biases in data collection represents a limitation.

Conclusions: In patients undergoing surgery for RCC, renal function should be preserved in order to improve cancer-related survival.

Patient summary: The relationship between renal function and probability of dying due to renal cancer is complex. The present study found a correlation between glomerular filtration rate and cancer specific mortality that could reconsider the oncological role of renal function in patients undergoing surgery for renal cancer.
\end{abstract}

(C) 2018 European Association of Urology. Published by Elsevier B.V. All rights reserved. 


\section{Introduction}

International guidelines [1,2] recommend partial over radical nephrectomy as the standard treatment for cT1 renal cell carcinoma (RCC) because of equivalent oncological outcomes [3] with superior preservation of renal function, postulated as beneficial to reduce cardiovascular mortality [4-6]. Thus, the indication to partial nephrectomy is progressively expanding even if the trade-off between advantages and surgical morbidity remains under debate [7].

The hypothesis that the amount of renal function could influence oncological outcomes is poorly intuitive and still neglected to date. However, at careful scrutiny within nephrology literature, it emerges that several large longitudinal population studies identified a linear inverse relationship between estimated glomerular filtration rate (eGFR) and cancer incidence or mortality [8-12].

If confirmed, this association would be paramount in patients with RCC, considering that surgical treatment directly impacts renal function. The present study analyzed a large comprehensive dataset to investigate this hypothesis.

\section{Patients and methods}

Five academic tertiary institutions collected full data on patients who consecutively underwent surgery for CT1-2, N0, and M0 (AJCC/TNM 2017) RCC from 1990 to 2015.

Indication to partial nephrectomy was clinically organ-confined tumor deemed as technically resectable by an experienced surgeon at each institution. During the study period, the tumor size cutoff to attempt partial nephrectomy was progressively raised from $2-3 \mathrm{~cm}$ to $7 \mathrm{~cm}$, in accordance with the contemporary guidelines [1,2].

The eGFR was evaluated by the Chronic Kidney Disease Epidemiology Collaboration (CKD-EPI) equation [13] at baseline, 12 mo after surgery, and last available follow-up, to account for complete stabilization from the surgical insult.

Patient's life status was documented by planned controls, and causes of death were reported as related to RCC or due to other causes; incomplete information was supplemented by phone interviews or by consulting hospital and administrative registries.

\subsection{Statistical analysis}

The relationship between eGFR and cancer-specific mortality (CSM) was analyzed in a competing-risk framework [14], accounting for death from other causes. Subdistribution hazard ratio (SHR) is the ratio of the instantaneous risk at time $t$ of having the event of death of two groups and can be interpreted as hazard ratios (HRs) of the Cox model. As readings were taken at three time points, two of which were fixed (baseline and 12 mo postoperatively) and the third one (last functional follow-up) scattered along the timeline, eGFR was first incorporated as a time-dependent covariate in an extended Fine and Gray model [15] by expanding data from one record per patient to one record per time interval per patient. The results of the model were compared with those of a landmark analysis [16] investigating the association between eGFR and CSM by setting three landmark time points (baseline, $12 \mathrm{mo}$, and last functional follow-up) and estimating a standard Fine and Gray model for each one with eGFR as a fixed-time covariate. Selection of predictors for all models was performed by estimating variable importance by the random survival forest method and then by backward elimination [17].
Exploratory graphs were drawn to visualize the relationship between eGFR and CSM: scatterplots with smoothed curves estimated by local regression (LOESS) suggested to model such a relationship by a linear spline, that is, a continuous piecewise linear function with two lines joined by a knot, the value of which was estimated by two different statistical methods [18,19]. Then, final competing-risk multivariable models were built taking into account the piecewise relationship.

As confirmatory analysis, the relationship between eGFR and CSM was investigated by the "joint modeling" [20] approach, which incorporated eGFR as a time-dependent covariate and accounted for the differences between pre- and postoperative periods by joining a linear mixed-effect model for longitudinal repeated measurements of eGFR and a Cox model with CSM as outcome, with a binary pre/postcovariate that differentiated between eGFR measurements before or after surgery.

A $p$ value of $<0.05$ was considered statistically significant. Calculations were done with Stata 13 (StataCorp, College Station, TX, USA) and R (version 3.4.4; R Foundation for Statistical Computing, Vienna, Austria).

\section{Results}

The dataset included 3457 patients with cT1a/T1b/T2, N0, M0 RCC; 1335 (39\%) underwent radical nephrectomy and 2122 (61\%) partial nephrectomy (Table 1).

Median functional follow-up was 61 mo (interquartile range [IQR] 24-118) with 7381 eGFR assessments recorded (2939 at baseline and 4442 after a minimum of 12 mo from surgery); $40 \%, 35 \%$, and $26 \%$ of patients had three, two, and one measurement, respectively; $66 \%$ had both pre- and postoperative measurements, $19 \%$ the preoperative one only, and $14 \%$ the postoperative one only. Median eGFR was 78.5 (IQR 65.4-92.6) $\mathrm{ml} / \mathrm{min}$ at baseline, 71.4 (IQR 54.689.6 ) $\mathrm{ml} / \mathrm{min}$ after $12 \mathrm{mo}$, and 64.4 (IQR $51.5-79.3$ ) $\mathrm{ml} / \mathrm{min}$ at the last functional follow-up; the respective percentages of patients at chronic kidney disease (CKD) stage $\geq 3$ were $16 \%, 33 \%$, and $38 \%$.

Overall 3008 patients were alive without evidence of recurrence (follow-up >60 mo in 48\%); 128 died due to RCC and 278 due to other causes. The estimated overall survival rates at 60 and 120 mo were $93 \%$ and $84 \%$, respectively.

Table 2 reports competing-risk univariate analysis: CSM was found to be significantly associated with age, gender, and descriptive tumor features (staging, grading, clear cell histology, and necrosis). Notably, eGFR and the presence of stage 3 CKD were found to be significantly related to CSM, with SHRs of 0.70 and 1.95 , respectively.

The relationship between the CSM and eGFR based on all, baseline, 12-mo, and last available functional dataadjusted for gender, age, pT stage, grading, and type of surgery-was graphically investigated as exemplified by Figs. 1 and 2, referring to the analysis of the whole data with eGFR as time dependent. As anticipated, this preliminary evaluation was suggested to model the relationship by a continuous piecewise linear function in which two lines are joined by a knot. The two statistical methods applied for knot location $[18,19]$ were consistent in finding a cutoff of $65 \mathrm{ml} / \mathrm{min}$ with eGFR as a timedependent covariate, and 85,60 , and $65 \mathrm{ml} / \mathrm{min}$ with eGFR as a time-fixed covariate at baseline, $12 \mathrm{mo}$, and last functional follow-up, respectively. 
Table 1 - Baseline characteristics of the study cohort

\begin{tabular}{|c|c|c|}
\hline Feature & & Value \\
\hline Age (yr) & Median (IQR) & $62.2(53.0-70.7)$ \\
\hline \multirow[t]{2}{*}{ Gender } & M & $2329(67 \%)$ \\
\hline & $\mathrm{F}$ & $1128(33 \%)$ \\
\hline Creatinine at diagnosis (mg/dl) & Median (IQR) & $0.9(0.8-1.1)$ \\
\hline Body mass index & Median (IQR) & $25.7(23.4-28.1)$ \\
\hline \multirow[t]{3}{*}{ Hypertension } & No & $2041(63 \%)$ \\
\hline & Controlled & $1121(34 \%)$ \\
\hline & Uncontrolled & $106(3 \%)$ \\
\hline \multirow[t]{2}{*}{ Diabetes } & No & $2992(91 \%)$ \\
\hline & Yes & $302(9 \%)$ \\
\hline \multirow[t]{3}{*}{ Charlson comorbidity index } & 0 & $1625(53 \%)$ \\
\hline & 1 & $651(21 \%)$ \\
\hline & $\geq 2$ & $801(26 \%)$ \\
\hline \multirow[t]{3}{*}{ Symptoms at diagnosis } & No & $2328(73 \%)$ \\
\hline & Local & $810(25 \%)$ \\
\hline & Systemic & $48(2 \%)$ \\
\hline \multirow[t]{3}{*}{ Clinical T stage } & T1a & $2048(59 \%)$ \\
\hline & T1b & $1043(30 \%)$ \\
\hline & $\mathrm{T} 2$ & $361(11 \%)$ \\
\hline \multirow[t]{2}{*}{ Surgery type } & Partial nephrectomy & $2122(61 \%)$ \\
\hline & Radical nephrectomy & $1335(39 \%)$ \\
\hline \multirow[t]{3}{*}{ Ischemia (partial nephrectomy) } & No & $991(40 \%)$ \\
\hline & Warm & $1449(59 \%)$ \\
\hline & Cold & $20(1 \%)$ \\
\hline \multirow[t]{2}{*}{ Blood transfusion } & No & $2730(84 \%)$ \\
\hline & Yes & $511(16 \%)$ \\
\hline \multirow[t]{4}{*}{ Complications (Clavien-Dindo) } & No & $2610(82.6 \%)$ \\
\hline & Minor (1-2) & $440(13.9 \%)$ \\
\hline & Major (3-4) & $98(3.1 \%)$ \\
\hline & Death (5) & $12(0.4 \%)$ \\
\hline Tumor diameter $(\mathrm{cm})$ & Median (IQR) & $3.7(2.5-5.0)$ \\
\hline \multirow[t]{5}{*}{ Pathological T stage } & T1a & $1850(58 \%)$ \\
\hline & T1b & 779 (24\%) \\
\hline & $\mathrm{T} 2 \mathrm{a}$ & $189(5 \%)$ \\
\hline & $\mathrm{T} 2 \mathrm{~b}$ & $27(1 \%)$ \\
\hline & T3-T4 & $339(11 \%)$ \\
\hline \multirow[t]{3}{*}{ Pathological N stage } & NO & $1141(33 \%)$ \\
\hline & $\mathrm{Nx}$ & $2300(66 \%)$ \\
\hline & $\mathrm{N}+$ & $16(1 \%)$ \\
\hline \multirow[t]{5}{*}{ Histological subtype } & Clear cell & $2268(72.1 \%)$ \\
\hline & Papillary & $592(18.8 \%)$ \\
\hline & Chromophobe & $240(7.6 \%)$ \\
\hline & Collecting duct & $13(0.4 \%)$ \\
\hline & Others & $32(1.0 \%)$ \\
\hline \multirow[t]{2}{*}{ Fuhrman's grading } & $1-2$ & $2351(77 \%)$ \\
\hline & $3-4$ & $692(23 \%)$ \\
\hline Sarcomatoid dedifferentiation & & $75(2 \%)$ \\
\hline Necrosis & & $534(18 \%)$ \\
\hline Positive surgical margins & & $86(3 \%)$ \\
\hline
\end{tabular}

Final multivariable competing-risk models showed an inverse and linear relationship between eGFR and CSM only below such cutoffs. In particular, for every $10 \mathrm{ml} / \mathrm{min}$ of decrease in eGFR, the SHR was 1.25 (95\% confidence interval [CI] 1.07-1.44, $p=0.003)$ below $65 \mathrm{ml} / \mathrm{min}$ for timedependent eGFR, 1.16 (95\% CI 1.02-1.31, $p=0.028$ ) below $85 \mathrm{ml} / \mathrm{min}$ for eGFR at baseline, 1.44 (95\% CI 1.06-1.96, $p=0.02$ ) below $60 \mathrm{ml} / \mathrm{min}$ for eGFR at $12 \mathrm{mo}$, and $1.16(95 \%$ CI 1.01-1.33, $p=0.042$ ) below $65 \mathrm{ml} / \mathrm{min}$ for eGFR at the last functional follow-up (Table 3 and Supplementary Table 1).

A negative significant association between eGFR and CSM only below $65 \mathrm{ml} / \mathrm{min}$ (SHR $=1.39$ for a decrease in eGFR of $10 \mathrm{ml} / \mathrm{min}, 95 \% \mathrm{CI} 1.06-1.82, p=0.018)$ was confirmed by joint modeling (Supplementary Table 2).
Table 2 - Univariate estimation of the correlation between features of the cohort and cancer-specific mortality by a Fine and Gray's competing risk regression model

\begin{tabular}{|c|c|c|c|}
\hline Variable & SHR & $95 \% \mathrm{CI}$ & $p$ value \\
\hline Age (yr, continuous) & 1.38 & $1.15-1.67$ & 0.001 \\
\hline BMI (kg/m², continuous) & 0.78 & $0.60-1.01$ & 0.055 \\
\hline Tumor diameter (cm, continuous) & 1.61 & $1.46-1.78$ & $<0.001$ \\
\hline Female gender & 0.58 & $0.38-0.89$ & 0.01 \\
\hline Hypertension & 1.35 & $0.93-1.96$ & 0.1 \\
\hline Diabetes mellitus & 1.39 & $0.78-2.47$ & 0.26 \\
\hline \multicolumn{4}{|l|}{ Charlson comorbidity index } \\
\hline 0 & 1.00 & - & - \\
\hline 1 & 1.17 & $0.74-1.86$ & 0.5 \\
\hline$\geq 2$ & 1.39 & $0.92-2.10$ & 0.12 \\
\hline \multicolumn{4}{|l|}{ Clinical stage } \\
\hline T1a & 1.00 & - & - \\
\hline T1b & 2.92 & $1.86-4.61$ & $<0.001$ \\
\hline $\mathrm{T} 2$ & 5.92 & $3.66-9.57$ & $<0.001$ \\
\hline Clear cell histological subtype & 2.03 & $1.16-3.54$ & 0.013 \\
\hline High grading & 2.94 & $2.06-4.20$ & $<0.001$ \\
\hline Necrosis & 2.01 & $1.35-3.00$ & 0.001 \\
\hline Positive surgical margins & 1.63 & $0.40-6.75$ & 0.5 \\
\hline \multicolumn{4}{|l|}{ Pathological stage } \\
\hline T1a & 1.00 & - & - \\
\hline T1b & 2.61 & $1.55-4.40$ & $<0.001$ \\
\hline $\mathrm{T} 2$ & 4.74 & $2.61-8.62$ & $<0.001$ \\
\hline T3/4 & 7.95 & $4.80-13.15$ & $<0.001$ \\
\hline \multicolumn{4}{|l|}{ Type of surgery } \\
\hline Partial nephrectomy & 1.00 & - & - \\
\hline Radical nephrectomy & 4.75 & $2.83-7.97$ & $<0.001$ \\
\hline eGFR (ml/min, continuous) & 0.70 & $0.58-0.83$ & $<0.001$ \\
\hline CKD stage $\geq 3$ & 1.95 & $1.25-3.05$ & 0.003 \\
\hline $\begin{array}{l}\text { Continuous variables have been sta } \\
\mathrm{CI}=\text { confidence interval; } \mathrm{CKD}=\mathrm{chr} \\
\text { glomerular filtration rate; } \mathrm{SHR}=\mathrm{su}\end{array}$ & $\begin{array}{l}\text { Irdizec } \\
\text { kidn } \\
\text { tribut }\end{array}$ & hazard ratio & timated \\
\hline
\end{tabular}

The analysis was replicated by splitting the cohort according to the type of surgery: breakpoints at $65 \mathrm{ml} / \mathrm{min}$ were estimated for both the subgroups, and a linear and inverse relationship between eGFR and CSM was found only below this value (partial nephrectomy SHR 1.35 [95\% CI $1.02-1.92, p=0.038]$; radical nephrectomy SHR 1.23 [95\% CI 1.03-1.47, $p=0.026])$.

\section{Discussion}

The main finding of the present study was that renal function showed an independent relationship with CSM, after accounting for other strong prognostic factors and competitive causes of mortality. This relationship was modeled by a "piecewise" linear function with a cutoff of $65 \mathrm{ml} / \mathrm{min}$ considering all data, $85 \mathrm{ml} / \mathrm{min}$ for preoperative values only, and between 60 and $65 \mathrm{ml} / \mathrm{min}$ for postoperative values only. It is likely that renal function becomes a determinant of CSM only when a certain degree of impairment is exceeded, according to a "threshold effect." In other words, the amount of renal function over breakpoints could represent a functional reserve within which CSM seems to be uninfluenced by eGFR. Conversely, when the reserve is "exhausted", any further decline proportionally increases CSM. It is worth remarking that this behavior is typical also for "nononcological" detrimental effects due to renal failure 


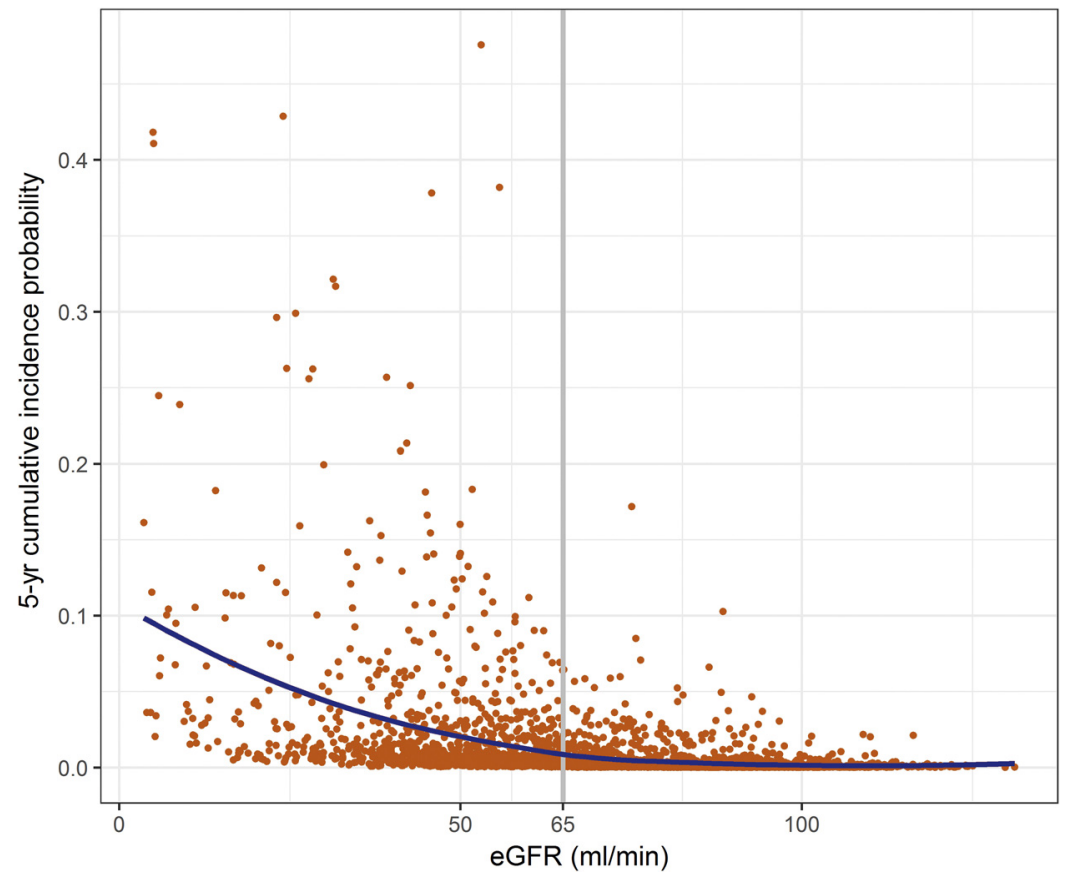

Fig. 1 - Relationship between eGFR and CIF-a measure of CSM in competing risk framework-at 60 mo: the smoothed curve estimated by local regression (LOESS) showed clear nonlinearity that could be approximated by a continuous piecewise linear function with two regression lines and one knot. The value of the $\mathrm{knot}$ was equal to $65 \mathrm{ml} / \mathrm{min}$, as determined by different methods. CIF = cumulative incidence function; CSM = cancer-specific mortality; eGFR = estimated glomerular filtration rate.

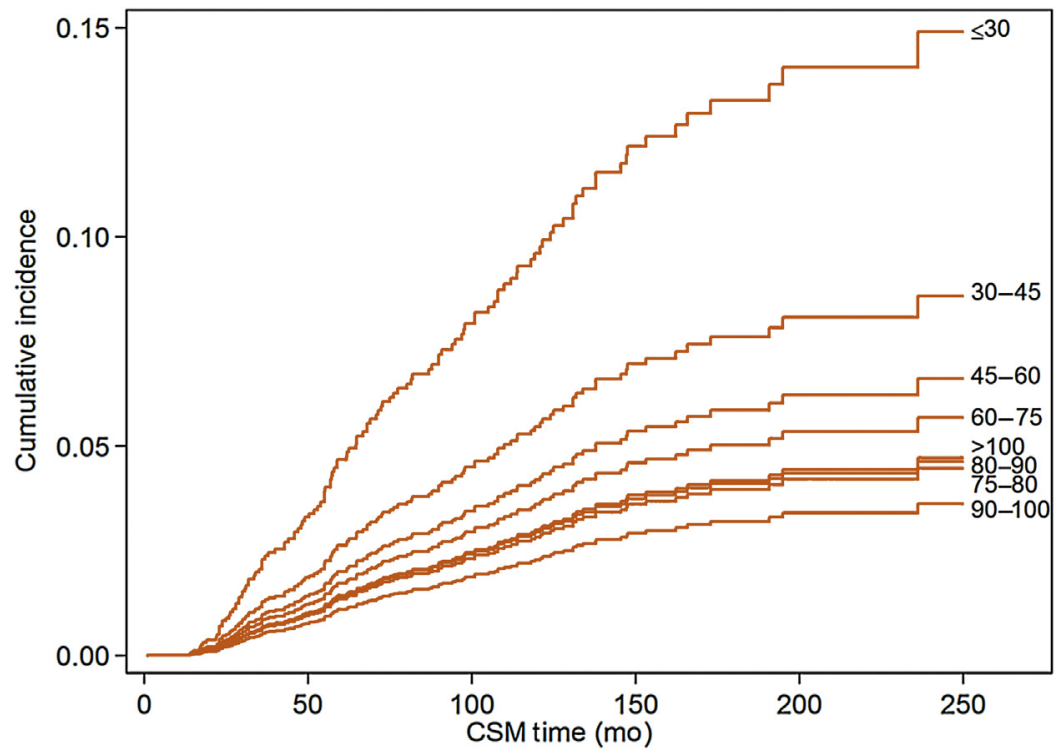

Fig. 2 - According to eGFR categorized into eight intervals, the graph represents the respective eight CIFs-a measure of CSM in competing risk framework-as estimated by a multivariable Fine and Gray model, setting the adjustment covariates gender, age, pT stage, and grading to their mean values. As for Fig. 1, the graph shows the existence of a nonlinear relationship between CSM and eGFR, linearly and inversely associated with values only below the breakpoints of $65 \mathrm{ml} / \mathrm{min}$. CIF = cumulative incidence function; $\mathrm{CSM}=$ cancer-specific mortality; eGFR = estimated glomerular filtration rate.

[4-6]. These results could influence surgical indication: as such, patients with baseline eGFR below $85 \mathrm{ml} / \mathrm{min}$ would start within the "risk" zone where any decrease of renal function would proportionally worsen CSM, unless postoperative eGFR was maintained over $60-65 \mathrm{ml} / \mathrm{min}$. Accordingly, partial nephrectomy should be preferred. Conversely, in patients for whom postoperative eGFR could be secured above $60-65 \mathrm{ml} / \mathrm{min}$, the role of surgery seems to be less determinant and they can be good candidates for radical nephrectomy as well. However, caution is needed considering that the prognostic relevance of preoperative eGFR raises the possibility that existing comorbidities could be equally or even more determinant than surgically induced CKD. 
Table 3 - Multivariable regression Fine and Gray extended model to estimate the relationship with cancer-specific mortality accounting for competing risks in pre- and postoperative periods

\begin{tabular}{|c|c|c|c|}
\hline Feature & SHR & $95 \% \mathrm{CI}$ & $p$ value \\
\hline Gender (female vs male) & 0.57 & $0.37-0.87$ & 0.01 \\
\hline Age (yr, continuous) & 1.01 & $0.99-1.03$ & 0.27 \\
\hline Type of surgery (radical vs partial nephrectomy) & 2.43 & $1.35-4.37$ & 0.003 \\
\hline pT (T1b vs T1a) & 1.86 & $1.07-3.24$ & 0.03 \\
\hline pT (T2 vs T1a) & 2.92 & $1.57-5.45$ & $<0.001$ \\
\hline pT (T3-T4 vs T1a) & 3.89 & $2.16-7.00$ & $<0.001$ \\
\hline Grading (high vs low) & 1.67 & $1.10-2.51$ & 0.015 \\
\hline eGFR below breakpoint (continuous, intervals of $10 \mathrm{ml} / \mathrm{min}$ ) & 1.25 & $1.07-1.44$ & 0.003 \\
\hline eGFR above breakpoint (continuous, intervals of $10 \mathrm{ml} / \mathrm{min}$ ) & 1.04 & $0.86-1.26$ & 0.72 \\
\hline
\end{tabular}

Large population [21] and retrospective hospital studies $[3-5,22]$ reported that renal function plays an independent role on non-cancer-related mortality. Definitely, the relationship between renal function and non-cancer-related mortality is robust and widely accepted, as also confirmed in our dataset by a multivariable Cox regression after accounting for several patient- and tumor-related confounders (HR for eGFR 0.98, 95\% CI 0.98-0.99, $p<0.001$; analysis not reported).

Conversely, at present, the urological community has omitted the hypothesis that the impairment of renal function could impact CSM, although there is evidence that CKD, also at an initial stage, may worsen the response against cancer. Indeed, large longitudinal population studies showed a linear inverse relationship between eGFR, incidence of cancer [7-11], and CSM [10,23,24], in particular for RCC. Mok et al [23] reported a linear inverse relationship between eGFR $<45 \mathrm{ml} / \mathrm{min}$ and CSM, especially for RCC, in 367392 patients followed for a median of 9.8 yr. Iff et al [24] found a linear inverse relationship between eGFR $<60 \mathrm{ml} / \mathrm{min}$ and CSM in 4077 patients followed for a median of $12.8 \mathrm{yr}$; this relationship was more robust for breast and urinary cancers and independent of competing causes of mortality. Weng et al [10] found an increased risk of CSM proportional to eGFR reduction in 123717 Taiwanese followed for a median of $7 \mathrm{yr}$; again, this effect was more pronounced for liver, kidney, and urinary tract cancers. In addition, one retrospective multi-institutional "urological" paper reported worse CSM after radical nephrectomy in 200 patients with preoperative CKD when compared with 600 matched controls [25].

With respect to the existing literature, the present study provides detailed information on treatments and pathological features, major determinants of CSM crucial to adequately conduct survival analysis. Renal function was longitudinally recorded during follow-up, and not only at baseline, in order to investigate whether CSM had a relationship with synchronous eGFR. Finally, statistical methods and prolonged follow-up allowed for the adjustment of the association between renal function and CSM for competitive causes of mortality, especially relevant in such population with low-risk renal cancer.
Comprehensive meta-analysis of retrospective observational studies showed that partial nephrectomy had lower CSM than radical nephrectomy, providing a $29 \%$ risk reduction in CSM (HR 0.71, $p<0.001$ ) [3] despite comparable tumor characteristics. Such a paradoxical association between most radical therapies and the worse oncological outcome was also found in our results, but no causality should be claimed since selection bias was not controlled due to the retrospective design of studies. Nevertheless, we attempted to mitigate this bias during the assessment of the eGFR/CSM relationship, including several tumor-related features in multivariable analyses, and also of the type of surgery per se. Further subgroup analysis confirmed our findings in patients submitted to partial or radical nephrectomy only. Ultimately, it seems that the relationship between eGFR and CSM was not influenced by tumorrelated features, explicitly reported or hidden behind the type of surgery.

Our findings pose some concerns regarding the results of previous retrospective studies comparing the oncological outcomes of partial versus radical nephrectomy, neglecting the role of renal function as a prognostic factor. The hypothesis that higher preservation of renal function could have impacted these results, by favoring the patients who underwent partial nephrectomy due to superior preservation of renal function, should be discussed. In opposition to these considerations, the randomized trial EORTC 30904 $[26,27]$ reported comparable CSM after partial and radical nephrectomy. Besides the numerous limitations of this trial [28], it should be remarked that oncological and functional follow-up periods were not congruent (median time 9.3 vs $6.7 \mathrm{yr}$ ) and that the analyses were not adjusted for competing causes of mortality, despite a large number of patients dying from non-cancer-related causes.

We acknowledge that the association of renal function with prognosis after cancer surgery is not intuitive and that the underlying causes are difficult to deduce. Nevertheless, in patients with decreased renal function, there is a complex set of disorders of the adaptive immune system that has been investigated in depth. Loss of renal function determines the onset of a vicious loop in which the immune system is both activated in a proinflammatory direction and suppressed, finally leading to uremia-related immune 
deficiency [29]. More extensively, CKD causes a preferential loss of number and function of lymphoid cells through the loss of thymic function, attrition of telomeres, and expanded memory T-cell population, which was compatible with the concept of premature immunological aging on the whole [30]. Finally, it could be hypothesized that preservation of renal function promotes physical and mental health status, and contributes to improving the host's defense against cancer.

The present study was not devoid of limitations. The first was the retrospective design with inherent biases in data collection, particularly concerning the proteinuria (the lack of this information compromised a more accurate estimation of renal damage) and the pattern of missing eGFR measurements. Second, as only cT1/2 renal masses were included as cases amenable for both partial and radical nephrectomy, for more advanced tumors the association eGFR/CSM might be masked by the prevailing role of pathological adverse features. Third, it cannot be excluded that functional impairment could be related to CSM just because a proxy of tumor volume and complexity, but due to the lack of a radiological review this issue cannot be addressed. Fourth, the long time span of the study impacted the surgical technique, with a shift toward minimally invasive approaches. Finally, we claim that the length of follow-up could be inadequate to clearly depict CSM in a cohort of patients mainly affected by low-risk tumors, burdened by a $5 \%$ risk of "very late" ( $>10 \mathrm{yr}$ ) recurrences [31].

\section{Conclusions}

The relationship found between eGFR and CSM indicates that renal function could have an oncological role in patients undergoing surgery for RCC. Whenever feasible, nephron-sparing approaches should be preferred, unless extirpative surgery could equally warrant the preservation of renal function above specific limits.

Author contributions: Alessandro Antonelli had full access to all the data in the study and takes responsibility for the integrity of the data and the accuracy of the data analysis.

Study concept and design: Antonelli, Minervini, Capitanio.

Acquisition of data: Furlan, Palumbo, Mari, Bertolo, Mantica, Larcher.

Analysis and interpretation of data: Antonelli, Sandri.

Drafting of the manuscript: Antonelli.

Critical revision of the manuscript for important intellectual content:

Terrone, Romagnani, Minervini, Capitanio, Mari, Bertolo.

Statistical analysis: Sandri.

Obtaining funding: None.

Administrative, technical, or material support: None.

Supervision: Simeone, Carini, Porpiglia, Montorsi, Bertini.

Other: None.

Financial disclosures: Alessandro Antonelli certifies that all conflicts of interest, including specific financial interests and relationships and affiliations relevant to the subject matter or materials discussed in the manuscript (eg, employment/affiliation, grants or funding, consultancies, honoraria, stock ownership or options, expert testimony, royalties, or patents filed, received, or pending), are the following: None.
Funding/Support and role of the sponsor: None.

Acknowledgments: The authors give special thanks to Mrs. Barbara Tucky for language revision.

\section{Appendix A. Supplementary data}

Supplementary material related to this article can be found, in the online version, at https://doi.org/10.1016/j. eururo.2018.07.029.

\section{References}

[1] Ljungberg B, Bensalah K, Canfield S, et al. EAU guidelines on renal cell carcinoma: 2014 update. Eur Urol 2015;67:913-24.

[2] Campbell SC, Novick AC, Belldegrun A, et al. Guideline for management of the clinical T1 renal mass. J Urol 2009;182:1271-9.

[3] Kim SP, Thompson RH, Boorjian SA, et al. Comparative effectiveness for survival and renal function of partial and radical nephrectomy for localized renal tumors: a systematic review and meta-analysis. J Urol 2012;188:51-7.

[4] Wu J, Suk-Ouichai C, Dong W, et al. Analysis of survival for patients with chronic kidney disease primarily related to renal cancer surgery. BJU Int 2018;121:93-100.

[5] Sun M, Trinh QD, Bianchi M, et al. A non-cancer-related survival benefit is associated with partial nephrectomy. Eur Urol 2012;61:725-31.

[6] Larcher A, Capitanio U, Terrone C, et al. Elective nephron sparing surgery decreases other cause mortality relative to radical nephrectomy only in specific subgroups of patients with renal cell carcinoma. J Urol 2016;196:1008-13.

[7] Van Poppel H, Joniau S, Albersen M. Nephron sparing for renal cell carcinoma: whenever possible? Eur Urol Focus 2016;2:656-9.

[8] Christensson A, Savage C, Sjoberg DD, et al. Association of cancer with moderately impaired renal function at baseline in a large, representative, population-based cohort followed for up to 30 years. Int J Cancer 2013;133:1452-8.

[9] Lowrance WT, Ordoñez J, Udaltsova N, Russo P, Go AS. CKD and the risk of incident cancer. J Am Soc Nephrol 2014;25:2327-34.

[10] Weng PH, Hung KY, Huang HL, Chen JH, Sung PK, Huang KC. Cancerspecific mortality in chronic kidney disease: longitudinal follow-up of a large cohort. Clin J Am Soc Nephrol 2011;6:1121-8.

[11] Vajdic CM, McDonald SP, McCredie MR, et al. Cancer incidence before and after kidney transplantation. JAMA 2006;296:2823-31.

[12] Shebl FM, Warren JL, Eggers PW, Engels EA. Cancer risk among elderly persons with end-stage renal disease: a population-based case-control study. BMC Nephrol 2012;13:65.

[13] Levey AS, Stevens LA. Estimating GFR using the CKD Epidemiology Collaboration (CKD-EPI) creatinine equation: more accurate GFR estimates, lower CKD prevalence estimates, and better risk predictions. Am J Kidney Dis 2010;55:622-7.

[14] Pintilie M. Competing risks. A practical perspective. Chichester, England: John Wiley \& Sons Ltd; 2006.

[15] Beyersmann J, Schumacher M. Time-dependent covariates in the proportional subdistribution hazards model for competing risks. Biostatistics 2008;9:765-76.

[16] Putter H. Handbook of survival analysis. Boca Raton, FL: Chapman \& Hall/CRC; 2013. p. 441-56, Chapter 21.

[17] Ishwaran H, Kogalur UB, Blackstone EH, Lauer MS. Random survival forests. Ann Appl Stat 2008;2:841-60. 
[18] Spiriti S, Eubank R, Smith P, Young D. Knot selection for least-squares and penalized splines. J Stat Comput Simul 2013;83:1020-36.

[19] Muggeo VMR. Estimating regression models with unknown breakpoints. Stat Med 2003;22:3055-71.

[20] Elashoff R, Li G, Li N. Joint modeling of longitudinal and time-toevent data. Boca Raton, FL: Chapman and Hall/CRC; 2016.

[21] Chronic Kidney Disease Prognosis Consortium, Matsushita K, van der Velde M, et al. Association of estimated glomerular filtration rate and albuminuria with all-cause and cardiovascular mortality in general population cohorts: a collaborative meta-analysis. Lancet 2010;375:2073-81.

[22] Kaushik D, Kim SP, Childs MA, et al. Overall survival and development of stage IV chronic kidney disease in patients undergoing partial and radical nephrectomy for benign renal tumors. Eur Urol 2013;64:600-6.

[23] Mok Y, Matsushita K, Sang Y, et al. Association of kidney disease measures with cause-specific mortality: the Korean Heart Study. PLoS One 2016;11:e0153429.

[24] Iff S, Craig JC, Turner R, et al. Reduced estimated GFR and cancer mortality. Am J Kidney Dis 2014;63:23-30.

[25] Kim YW, Kim WT, Yun SJ, et al. Preoperative chronic kidney disease status is an independent prognostic factor in patients with renal cell carcinoma. Ann Surg Oncol 2015;22:4098-103.
[26] Van Poppel H, Da Pozzo L, Albrecht W, et al. A prospective, randomized EORTC intergroup phase 3 study comparing the oncologic outcome of elective nephron-sparing surgery and radical nephrectomy for low-stage renal cell carcinoma. Eur Urol 2011;59:543-52.

[27] Scosyrev E, Messing EM, Sylvester R, Campbell S, Van Poppel H. Renal function after nephron-sparing surgery versus radical nephrectomy: results from EORTC randomized trial 30904. Eur Urol 2014;65:372-7.

[28] Thompson RH. Partial versus radical nephrectomy: the debate regarding renal function ends while the survival controversy continues. Eur Urol 2014;65:378-9.

[29] Kato S, Chmielewski M, Honda H, et al. Aspects of immune dysfunction in end-stage renal disease. Clin J Am Soc Nephrol 2008;3:1526-33.

[30] Betjes MG, Litjens NH. Chronic kidney disease and premature ageing of the adaptive immune response. Curr Urol Rep 2015; $16: 471$.

[31] Antonelli A, Furlan M, Sodano M, et al. Features, risk factors and clinical outcome of "very late" recurrences after surgery for localized renal carcinoma: a retrospective evaluation of a cohort with a minimum of 10 years of follow up. Int J Urol 2016;23:36-40.

\section{www.esukidneytransplant.org}

\section{1st ESU-ESTU Masterclass on Kidney transplant}

\section{5-16 November 2018, Madrid, Spain}

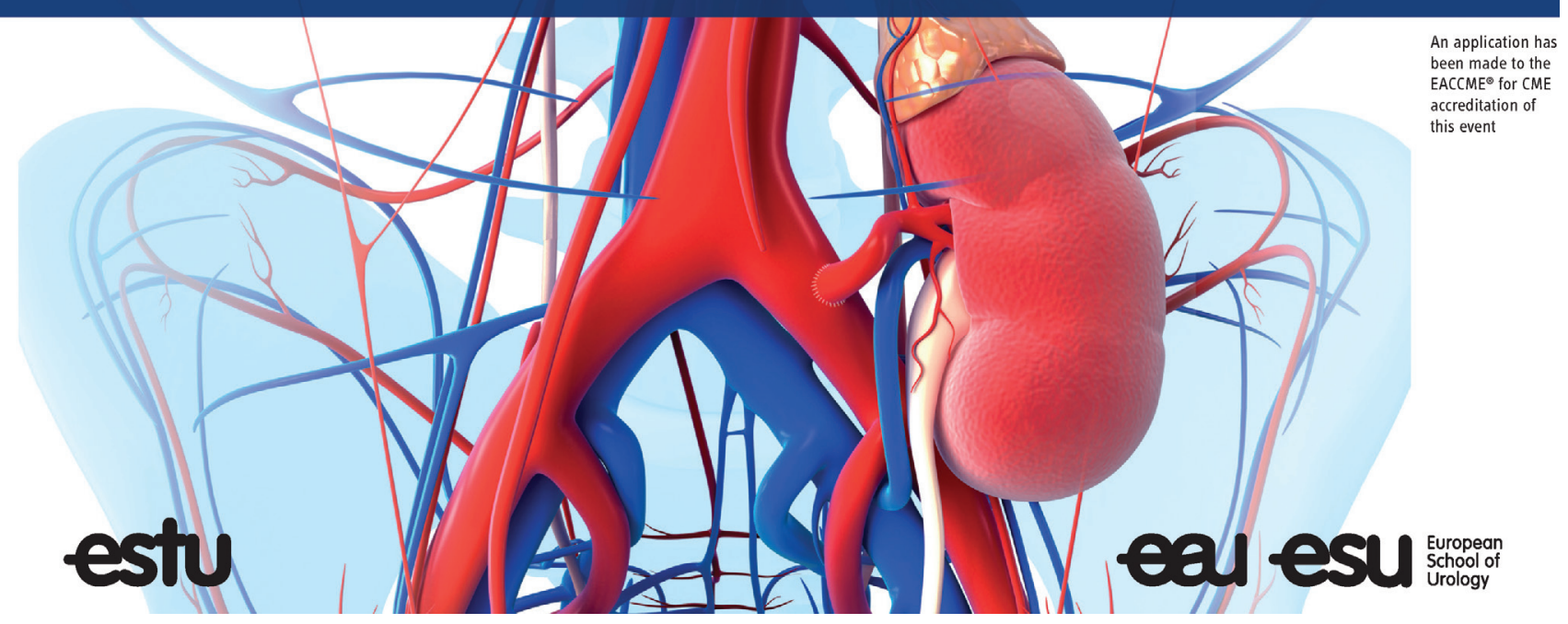

